\title{
Low Error Rate Data Transmission in Cognitive Radio Networks
}

\author{
A. NarendraKumar \\ Department of Electronics and Communication Engineering, V V college of Engineering, Tisaiyanvilai - 628656, \\ Tamilnadu, India \\ E-mail: nandhume@gmail.com
}

\section{ABSTRACT}

Cognitive Radio (CR) has become a hopeful technology to enhance the spectrum utilization through spectrum sharing between licensed user (primary user) and unlicensed user (secondary user). An vital rule mandated for the development of such frameworks are to develop solutions that don't require any changes to the existing primary user (PU) infrastructure. An Orthogonal Frequency Division Multiplexing (OFDM) is typically worn advancements in present wireless communication systems which has the possibility of fulfilling the demand for cognitive radios intrinsically or with slight changes. In this paper, Space time block codes is used. The various antennas used on both ends for trustworthy data broadcast and interference nulling schemes. These codes can accomplish full broadcast diversification determined via the number of broadcast antennas. The MIMO is worn for enhancing the power of a wireless link, to determine the issue for lower BER and achieve a superior performance.

Keywords: Cognitive Radio, Multiple input and Multiple output, Orthogonal Frequency Division Multiplexing, Space time block codes.

\section{INTRODUCTION}

The Cognitive Radios (CRs) are worn to increase the spectrum capability in wireless communications [4]. The Cognitive radio (CR) is a form of wireless communication in which the transceiver can cleverly identify the communication channels are in use and which are not and instantaneously move into unoccupied channels while avoiding from possessing ones. A License user which has higher priority called primary user. The user which has an absolute right to a certain spectrum band. The secondary user which is an unlicensed user, the user may access under certain negotiated conditions. Cognitive Radio (CR) permits the secondary user which is lower priority than primary user.

Cognitive Radio able to share the spectrum of the primary user to secondary user without affecting the primary user [8]. A spectrum hole [7] (or also called white space) is a band of frequencies assigned to a primary user, but at an appropriate time and exact geological location, the band is not being used by that user. Cognitive Radio uses Dynamic Spectrum Access [6] to allow the Secondary user to access the substantial spectrum holes or white spaces in the licensed spectrum bands.

By deploying multiple antennas at the transmitter and receiver, the transmit and receive diversification at the base station and the mobile unit as shown in Figure 1. The prospect of improving the reliabilty and throughput of the wireless communication link at no cost of additional spectrum.
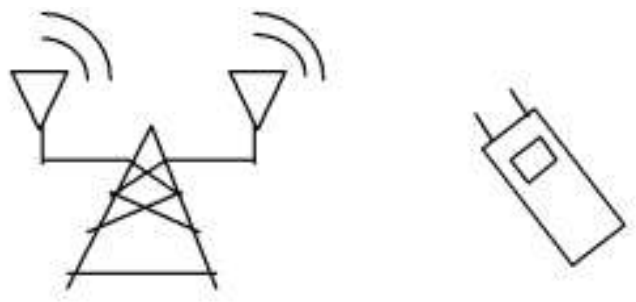

Fig 1: Transmit and Receive diversification in a MIMO channel

The space-time coding is the capacity to turn multipath propagation, impairment in single antenna wireless communications, into a benefit for multiple antenna systems by taking advantage of the arbitrary fading in expanding the transmission rate of the communication link as well as increases its reliability.

Spectrum sensing is the capability to calculate, sense and be aware of the parameters identified with the radio channel qualities, availability of spectrum and broadcast power, intrusion and noise, radio's operating atmosphere, user needs and applications, available networks and nodes, local policies and other operating limits. It is made across Frequency, Time, geological Space, Code and Phase.

\subsection{Related Work}

The end-to-end multipath RPC broadcast only access single relay route at a time, when concurrently transmitting numerous packets through numerous relay routes. This route is selected depends on the data sequence in the packet. 


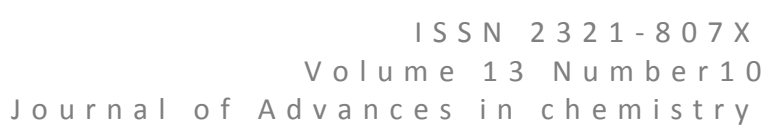

Hamming separations based RPC codes is utilized. RPC encodes the information packet in two courses, i.e., by exchanging the symbols with Quadrature Amplitude Modulation (QAM) and by utilizing a permutation cluster (PC) which comprises of an

arrangement of route indices [1]-[3]. The coded packet in each relay route is then broadened and sent towards the destination node. When the packet relaying between the nodes encounter the long end-to-end transmission latency, the packet is disposed, thus causing an erasure. Erasures happen because of deep fading or to the imperfection of spectrum sensing, which results in harsh intervention or collisions [2], when the coded packet is sent to a link occupied by the primary or other CR users.

On the encoding side, two transmit antennas are used as part of the multiple input and multiple output advances. In RPC (Route Permutation Codes), the error rate is not satisfied and low capacity. After that on the encoding side, the data packet is encoded by the STBC along the time coordinate [1].

The remaining sections of the paper are organized as follows: In section II, the System model is discussed. In Section III, we present the simulation results and discussion. The Conclusion is given in section IV.

\section{SYSTEM MODEL}

\subsection{Space Time Block Codes (STBC)}

In CRN, secondary users (SUs) can detect the channels of primary users (PUs) and which operates on the channel whose $\mathrm{PU}$ is absent.

Space-time block codes (STBC), the codes are orthogonal and can accomplish full broadcast diversification [5] specified via the number of broadcast antennas. Utilize various antennas on both sides for dependable data transmission. It is intended to accomplish the maximum diversification order on both sides. This has made space-time block codes most extensively utilized.

The capacity can be calculated by,

$\mathrm{C}_{\mathrm{n}}(\mathrm{nSNR}, \mathrm{nSample})=\log _{2}\left(\right.$ real $\left.\left(\left|1+\rho \leqslant \mathrm{H}^{\circ} * H\right|\right)\right)$

\subsection{Block Diagram}

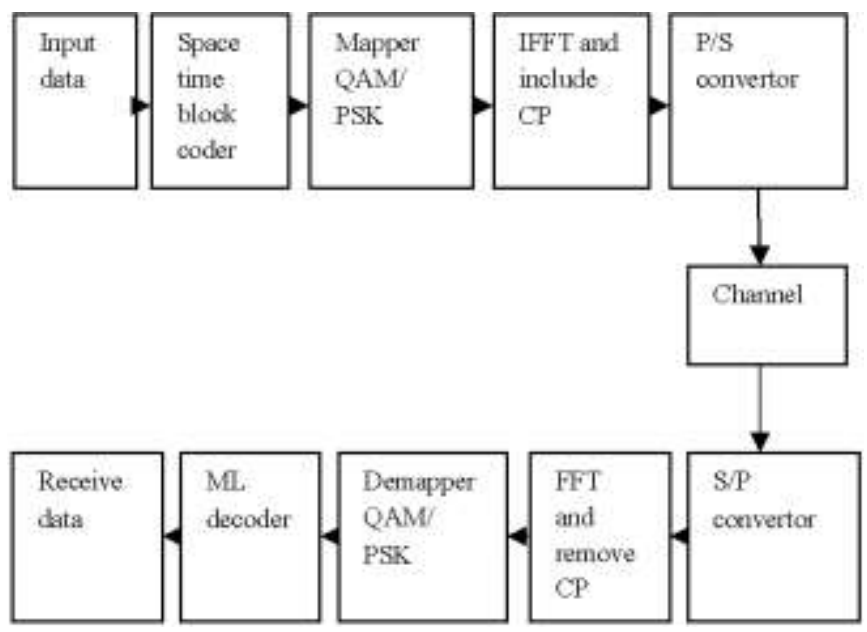

Fig 2: Block diagram

At OFDM transmitter, provide arbitrary input data for sending purposes. The input data is fed to the encoding. At the sending side, an STBC encoder scheme can accomplish a significant capacity gain or BER reduction. The Constellation mapper consists of a QAM modulator. It maps the arriving bits into independent sub-bearers. The IFFT changes over frequency domain constraints to a time domain. Cyclic Prefix (CP) entirely eliminate inter-symbol intrusion that happen because of Multiroute. Then the data are broadcast to a channel.

For channel, single route between the transmitter and the receiver occurs and an continuous attenuation and noise is considered. Additive white Gaussian noise (AWGN) is an essential noise model utilized as a part of Information theory to diminish the effect of many irregular procedures that happen in nature. It is a noise that is added to the OFDM modulated signal when it is passed through the channel.

At OFDM accepting side which taken its input from the transmitter at whatever its output is obtainable. The cyclic Prefix was further added at the sending side in order to avoid inter-symbol intrusion, therefore during the reception, it must be evacuated for any further processing of the inward signal. OFDM signals are obtained from the channel signal and are given to the FFT, 
which changes over them back to the frequency domain. The output data is given as input to the constellation de-mapper. The constellation de-mapper consists of a QAM demodulator. The ML (Maximum Likelihood) decoder [11] is utilized for decoding the output data.

\section{SIMULATION AND DISCUSSION}

For the simulation setup utilizing MATLAB [4], the performance of RPC and STBC in different stages are established.

The summary of parameters taken from [1] for simulation are given in Table 1.

TABLE 1 SUMMARY OF PARAMETERS

\begin{tabular}{|c|c|c|}
\hline Notation & Description & Specification \\
\hline \multirow[t]{3}{*}{$\rho$ (Rho) } & Route availability/ & 1. $0=$ no erasure \\
\hline & link availability/ & 2. $0.15=15 \%$ spectrum utilization \\
\hline & Spectrum utilization Range & $\begin{array}{l}\text { 3. } 0.3=30 \% \text { Doubled spectrum } \\
\text { utilization }\end{array}$ \\
\hline $\mathrm{R}$ & No. of users/ No. of Relay paths & 4.5 \\
\hline $\mathrm{N}=\mathrm{N}_{\mathrm{r}}$ & No. of opportunistic links in a path & 1,3 \\
\hline$W_{n}$ & Waiting period & ones $(1, R(1))^{\star} 6$ \\
\hline$d_{\min }$ & Minimum Hamming distance & $2,3,4$ \\
\hline$U_{R}$ & No. of CR users & \\
\hline $\mathrm{N}_{\mathrm{t}}$ & Transmit Antennas (RPC and STBC) & 2,2 \\
\hline $\mathrm{N}_{\mathrm{r}}$ & Receive Antennas (RPC and STBC) & 2,7 \\
\hline
\end{tabular}

The Node deployment of CR network is shown in Figure 3. This is designed with 4 numbers of relay node's connection between source and destination with 4 numbers of relay paths for reference.

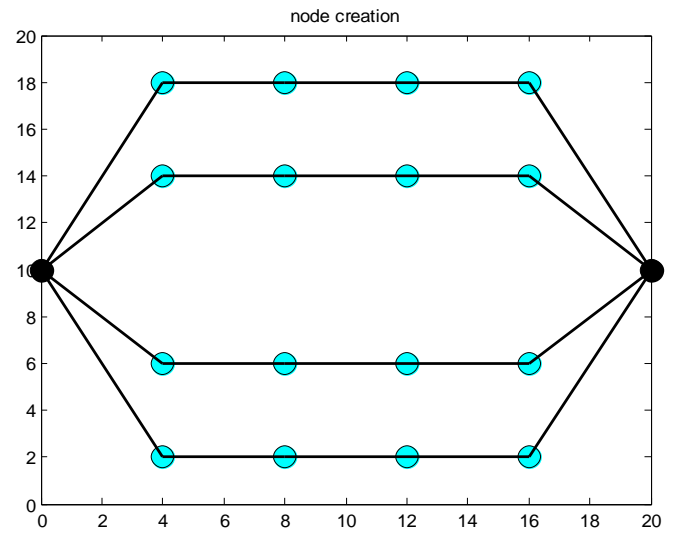

Fig 3: Node deployment for CR Network

The performance of RPC and STBC without considering the broadcast outage (erasure) is shown in Figure 4. The error rate caused by noise variation is analyzed with $\rho=0$, which implies that the links are always accessible. The BER testing of a variety of parameters of the RPC and STBC are compared. From this outcome, STBC conveys low error rate. 


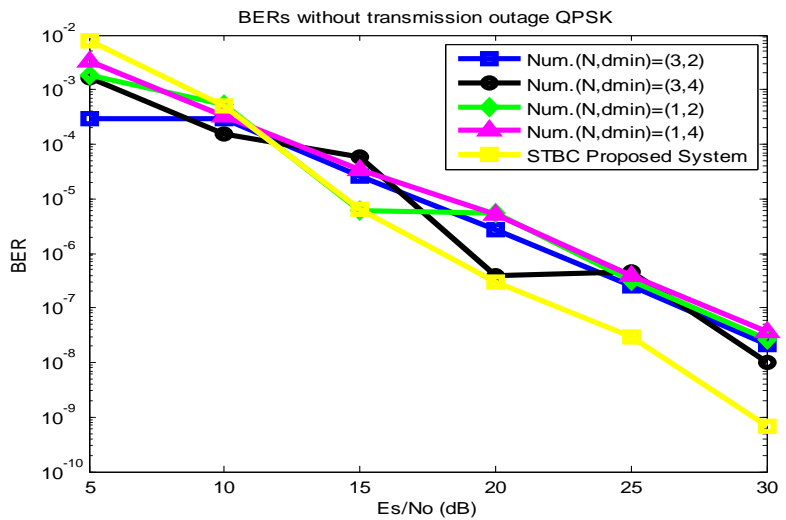

Fig 4: BER performance of RPC and STBC without erasure

The performance of RPC and STBC with erasure channels is shown in Figure 5. The BER testing of an assortment of parameters of the RPC and STBC are analyzed. From this outcome, contrasted with RPC, STBC conveys low error rate.

The performance of RPC and STBC in the multiuser situation is shown in Figure 6. The BER testing of an assortment of parameters of the RPC and STBC are compared. If the user increases, STBC performs low BER contrasted with RPC under the multiuser situation

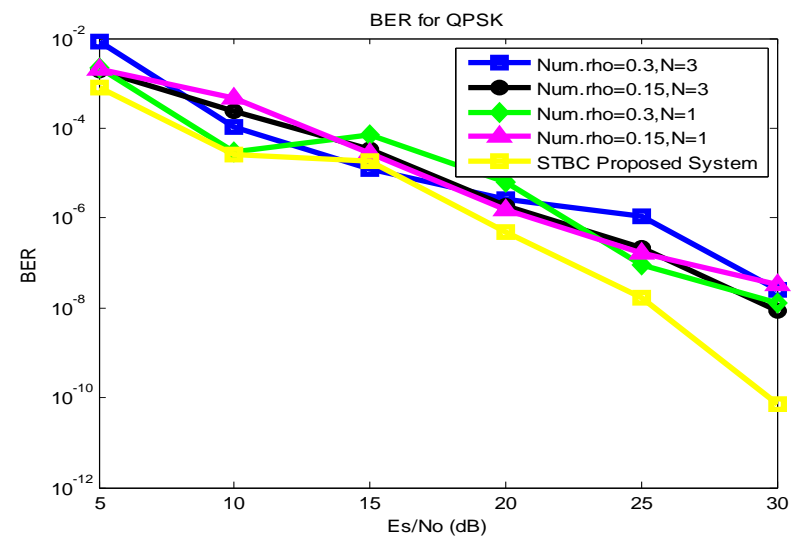

Fig 5: BER Performance of RPC and STBC with erasure

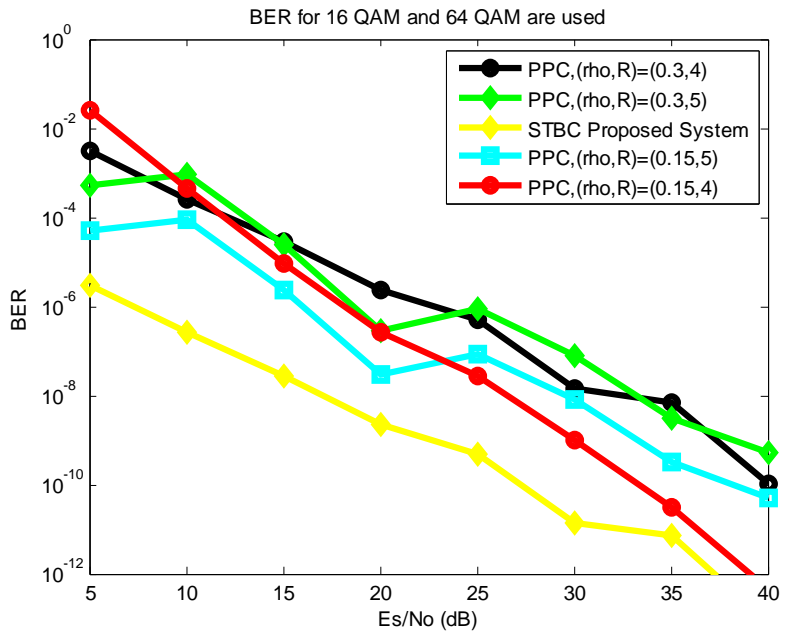

Fig 6: BER Performance of RPC and STBC under multiuser situation 


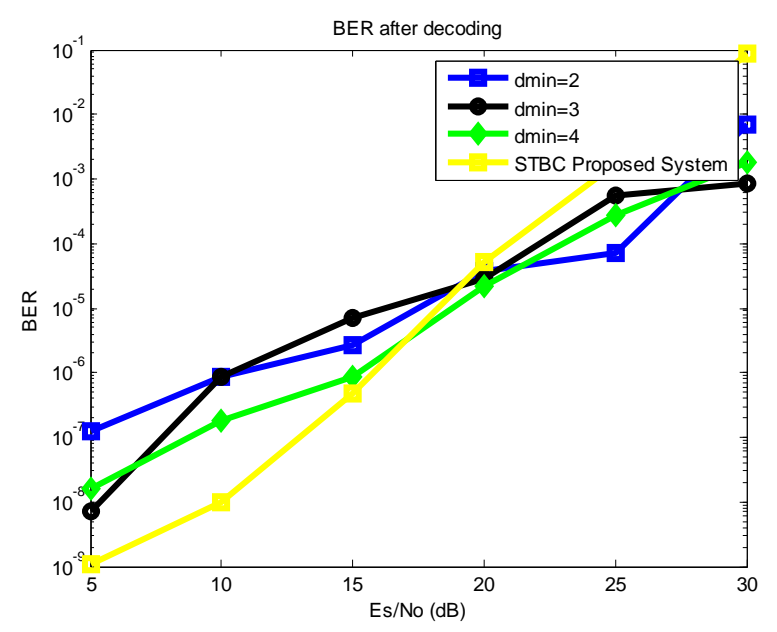

Fig 7: BER Performance of RPC and STBC utilizing ML Decoder

The error rate performance of RPC and STBC after decoding is shown in Figure 7.

The capability of RPC and STBC under multiuser situation is shown in Figure 8. For upgrading the capacity, MIMO is utilized. From this outcome, STBC conveys high capability contrasted with RPC.

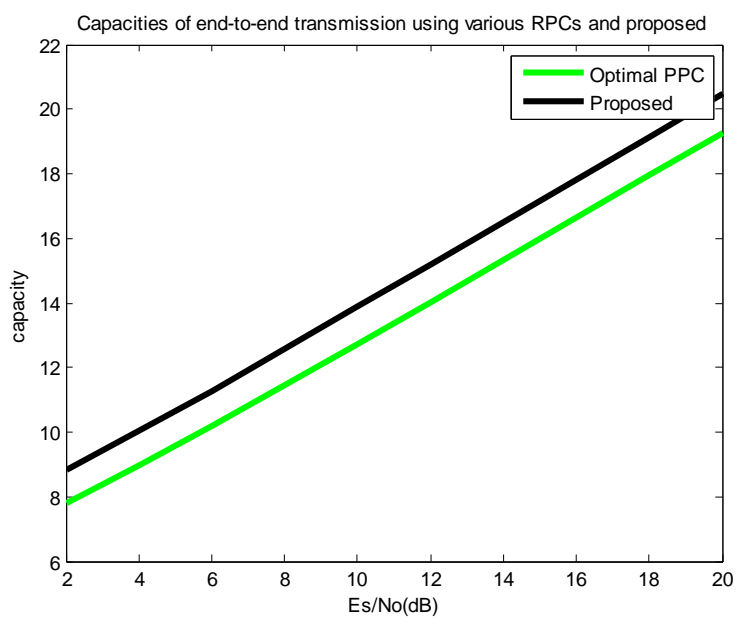

Fig 8: Capabilities of RPC and STBC under multiuser situation

\section{CONCLUSION}

In this paper, the STBC-OFDM scheme for an end to end broadcast in CRN's was proposed. These codes make utilization of various antennas that permit a system to have superior performance in a vanishing atmosphere. Amplify and forwarding diversification mechanism was utilized. We have demonstrated this at various stages and get superior performance in CRN with low BER. Likewise, a decoding approach Maximum Likelihood (ML) decoder that it is efficient in decoding to perform superior in the multiuser situation. To accomplish the low broadcast power through the broadcast period, the proposed technique is recommended for further usage in future.

\section{REFERENCES}

1. Lai, I. W., Lee, C. H, Chen, K. C and Bigleiri, E. 2015. Path-Permutation Codes for End-to-End Transmission in Ad Hoc Cognitive Radio Networks IEEE Transactions on wireless communications, vol. 14, no. 6, pp. 3309-3321 2015.

2. Lai, I. W., Chen, C. L., Lee, C. H., Chen, K. C., and Biglieri, E. 2014 End-to-end virtual MIMO Transmission in ad hoc cognitive radio networks IEEE Transactions Wireless Communication, vol. 13, no. 1, pp. 330-341.

3. Chen, K. C., and Lien, S. 2013 Machine-to-machine communications: Technologies and challenges Ad Hoc Network., vol. 18, pp. 3-23. 
Volume $13 \mathrm{Number} 10$

Journal of Advances in chemistry

4. MATLAB link: https://in.mathworks.com/

5. Bhattacharya, P. P., Khandelwal, R., Gera, R., and Agarwal, A. 2011 Smart Radio Spectrum Management for Cognitive Radio", International Journal of Distributed and Parallel Systems (IJDPS), Vol.2, No.4, pp.12-24

6. Das, P., Chatterjee, S., and Ghosh, G. 2014 "Cognitive Radio And Dynamic Spectrum Access - A Study", International Journal of Next-Generation Networks (IJNGN), Vol.6, No.1,

7. Rekha, P.R., and Varghese, J. 2016 A Study on Space Time Block Code and Space Frequency Block Code with MIMOOFDM in wireless communication systems International Journal of Innovative research in computer and communication Engineering, Vol. 4, pp. 13305-13310.

8. Haykin, S. 2005 Cognitive Radio: Brain-empowered Wireless Communications IEEE Journal on Selected Areas in Communication., Vol. 23, No. 2, pp. 201-220.

9. Raghuwanshi, S. and Barde, C. 2013 A Survey of Cognitive Radio Network Techniques and Architecture International Journal of Innovative Research in Engineering \& Multidisciplinary Physica I Sciences (IJIRMPS), Vol. 1, Issue 1, pp. 20-24, October 2013.

10. Rappaport, T. S. 2002 Wireless Communications: Principles and Practice, Pearson Education, Inc., Prentice Hall, 2 nd Edition, 2002.

11. Tarokh, V., Jafarkhani, H., and Calderbank, A. R. 1999 Space-Time Block Codes from Orthogonal Designs IEEE Transactions on Information Theory, Vol. 45, No. 5.

\section{Biography}

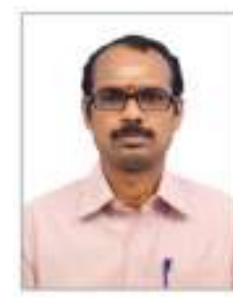

Dr. A. NarendraKumar, completed his Ph.D. in Network systems from Anna University, Chennai, India in 2015. He is currently working as a Professor, Department of Electronics and Communication Engineering, V V College of Engineering, Tisaiyanvilai, Tamil Nadu, India. His current research interest in Adhoc Network and fuzzy logics. He has published 5 research articles in various peer reviewed international journals and participated in various national and international conferences.

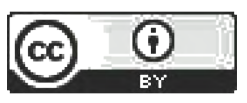

This work is licensed under a Creative Commons Attribution 4.0 International License. 